\title{
REFERENCE OBJECTS-BASED REAL ESTATE VALUATION WITH MDAHP
}

\author{
Mirosław Dytczak* \\ Faculty of Civil Engineering and Environmental Engineering \\ Białystok University of Technology \\ Białystok, Poland \\ E-mail: mdytczak@gmail.com \\ Grzegorz Ginda \\ Faculty of Material and Environment Sciences \\ University of Bielsko-Biała \\ E-mail: gg.ginda@gmail.com
}

\begin{abstract}
Official real estate valuation procedures allow a valuator to use experience without limits. A valuator therefore usually applies features of real estate objects which have been valuated already to evaluate features of a valuated real estate object. Some real estate features are intangible. It is therefore important to assess them properly to ensure reliable valuation results. Application of Multiple Dominant AHP is proposed with this regard. It facilitates making justified evaluations while still allowing to utilise valuator's experience and providing reliable valuation results reliable. A sample analysis is also included.
\end{abstract}

Keywords: real estate, valuation, reliability, experience, MDAHP

\section{Introduction}

Real estate valuation process depends on application of official procedures. Existing valuation rules require including all essential factors which influence a real estate value. Valuation deals with different factors including intangibles. Intangibles are usually dealt with by licensed valuators using intuition and personal experience. Exclusive application of intuition and experience makes real estate valuation process transparency and outcomes susceptible to suspicion with regard to fairness and reliability. Application of appropriate approach is therefore welcome to eliminate concerns due to intuitive and experience-based including of intangibles.

Traditional AHP methodology is well suited to decision support in the case of relative pair-wise comparisons. There appear situations, however, when considered decision problem model components need to be compared using absolute basis which corresponds to context nature of available information. Such situation undoubtedly pertains to real estate valuation. Licensed valuators use personal experience which results from results of past valuations. A valuator therefore evaluates real estate objects using features of known real estate objects he or she dealt with in the past. Such real estate objects can be therefore considered reference objects for valuation of other real estate objects.

It is possible, however, to take advantage of AHP benefits related to simplicity of data acquiring, data processing and including intangibles while considering template objects. Dominant AHP (DAHP) invented by Kinoshita and Nakanishi (1999) can be applied in this regard.

\footnotetext{
${ }^{*}$ Corresponding author
} 
The paper is continuation of a work (Dytczak et al., 2009) which deals with AHP application to real estate object valuation. DAHP is applied instead this time, however. DAHP calculus is therefore presented in the next section. Sample application for real estate object valuation is also included to illustrate DAHP application rules.

\section{Dominant AHP}

The main difference between DAHP and traditional AHP comes from a way evaluated objects are compared. Features of objects are compared relative to features of reference objects called regulatory decision alternatives (regulatory alternatives for short). Multiple regulatory alternatives can be also applied. DAHP becomes Multi-Dominant AHP (MDAHP) in such the case.

\subsection{The single regulatory object case}

Application of $K$ evaluation criteria results in the following formula for overall evaluation $\overline{v_{i}}$ of the i-th consecutive decision alternative out of $n$ considered alternatives:

$$
\overline{v_{i}}=\sum_{k=1}^{K} v_{i k},
$$

where $v_{i k}$ denotes partial evaluation of the i-th consecutive decision alternative with regard to the k-th consecutive criterion.

Let's consider $\mathrm{D}$ a set of evaluated objects and $r$ to being a single regulatory alternative:

$$
r \in D \text {. }
$$

Here is the DAHP procedure:

1. Partial evaluation of considered objects with regard to each criterion $(i=1,2 \ldots n, j=1,2 \ldots K)$ :

$$
\underset{i}{\forall} \underset{k}{\forall} \quad u_{i k}=\alpha_{k} v_{i k}
$$

where $\alpha_{k}$ is a parameter.

Partial evaluations data make creation of the following matrix possible:

$$
\mathbf{A}^{\prime}=\left[\begin{array}{cccc}
u_{11} & u_{12} & \ldots & u_{1 K} \\
u_{21} & u_{22} & \ldots & u_{2 K} \\
\vdots & \vdots & \ddots & \vdots \\
u_{n 1} & u_{n 2} & \ldots & u_{n K}
\end{array}\right]
$$

2. Estimation of partial relative evaluation $u_{i k}^{(r)}$ for each considered object according to each evaluation criterion:

$$
\underset{k=1 \ldots K}{\forall} u_{i k}^{(r)}=\frac{u_{i k}}{u_{r k}}
$$

Estimation of normalised weights for the criteria $w_{k}^{(r)}$ that correspond to the regulatory alternative $r$ : 


$$
\sum_{k=1}^{K} w_{k}^{(r)}=1
$$

3. Overall evaluation of considered decision alternatives:

$$
\underset{i}{\forall} t_{i}=\sum_{k=1}^{K} w_{k}^{(r)} u_{i k}^{(r)} .
$$

The formula (7) can be rewritten in the following way:

$$
\mathbf{T}=\overline{\mathbf{A}}^{(r)} \mathbf{w}^{(r)},
$$

where: vector $\mathbf{w}^{(r)}$ contains normalised weights of evaluation criteria:

$$
\mathbf{w}^{(r)}=\left[\begin{array}{llll}
w_{1}^{(r)} & w_{2}^{(r)} & \ldots & w_{K}^{(r)}
\end{array}\right]^{\mathrm{T}} .
$$

and $\overline{\mathbf{A}}^{(r)}$ denotes the following matrix:

$$
\overline{\mathbf{A}}^{(r)}=\left[\begin{array}{cccc}
u_{11}^{(r)} & u_{12}^{(r)} & \ldots & u_{1 K}^{(r)} \\
u_{12}^{(r)} & u_{22}^{(r)} & \ldots & u_{2 K}^{(r)} \\
\vdots & \vdots & \ddots & \vdots \\
u_{n 1}^{(r)} & u_{n 2}^{(r)} & \ldots & u_{n K}^{(r)}
\end{array}\right]
$$

Components of the matrix correspond to evaluation of decision alternatives relative to the regulatory alternative:

$$
\bar{a}_{i k}^{\prime(r)}=\frac{a_{i k}^{\prime}}{a_{r k}^{\prime}}=u_{i k}^{(r)} .
$$

Let's notice that both overall evaluation $t_{r}$ and partial evaluations $u_{r k}$ for the regulatory alternative are equal to 1 . Overall and partial evaluations of other decision making alternatives are therefore related to the regulatory alternative. Criteria in DAHP are also evaluated relative to the regulatory alternative.

Differences between DAHP and AHP are illustrated in Fig.1 which corresponds to evaluation of real estate object N. Three evaluation criteria are denoted by R, O and E. Symbols 14, 13, 4, 3, 8 correspond to decision alternatives - real estate objects of known values. The real estate object 4 is assumed the regulatory alternative in the case of the DAHP application. Continuous line corresponds to arcs which denote direct influence of model components while dashed line expresses indirect influence. It is evident that DAHP problem model includes influence feedback between components while traditional AHP problem model doesn't allow such relation at all. 


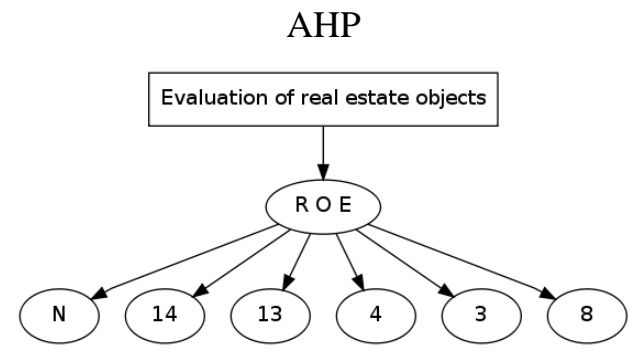

DAHP

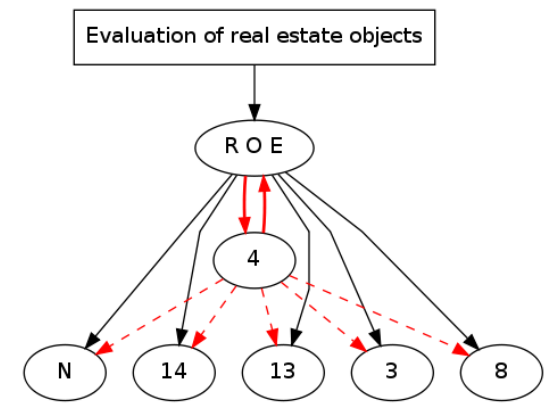

MDAHP

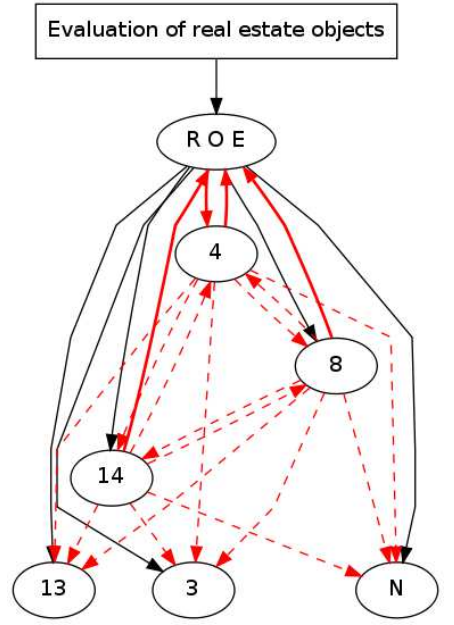

Fig.1. Differences between AHP, DAHP and MDAHP

\subsection{The case of multiple regulatory alternatives}

Multi-Dominant AHP is based on the similar assumptions as DAHP is. This time, however, we have got several regulatory alternatives which belong to a subset $\mathrm{R}$ of all decision making alternatives:

$$
R \subset A .
$$

Differences between MDAHP and DAHP are presented in Fig.2. It is evident that multiple regulatory alternatives influences complexity of applied problem model considerably.

We must be aware that application of multiple regulatory alternatives results in a need for adjustment of overall evaluations of objects relative to different regulatory alternatives. Co-ordination of weight values for evaluation criteria obtained relative to regulatory alternatives helps with this regard. Adjusted weights for evaluation criteria should satisfy the following condition:

$$
\underset{r, r^{\prime} \in R}{\forall} \quad w_{k}^{(r)}=\frac{\frac{w_{k}^{\left(r^{\prime}\right)} v_{r k}}{v_{r^{\prime} k}}}{\sum_{j=1}^{K} \frac{w_{j}^{\left(r^{\prime}\right)} v_{r j}}{v_{r^{\prime} j}}} .
$$


A step-wise concurrent convergence method (CCM) proposed by Kinoshita et al. (2002) makes such adjustment of criteria weights possible. It is based on application of the following recurrent formula:

$$
\underset{r \in R}{\forall} \quad \mathbf{w}^{(r)}=\frac{1}{|R|} \sum_{r^{\prime} \in R} \frac{\mathbf{A}^{(r)}\left(\mathbf{A}^{\left(r^{\prime}\right)}\right)^{-1} \mathbf{w}^{\left(r^{\prime}\right)}}{\mathbf{e}^{\mathrm{T}} \mathbf{A}^{(r)}\left(\mathbf{A}^{\left(r^{\prime}\right)}\right)^{-1} \mathbf{w}^{\left(r^{\prime}\right)}},
$$

where: $\mathbf{A}^{(r)}$ denotes diagonal matrix consisting partial evaluations obtained for the regulatory alternative $r$ with regard to the consecutive criteria: $a_{r k}(k=1,2 \ldots K)$ :

and $\mathbf{e}$ is the following vector:

$$
\mathbf{A}^{(r)}=\left[\begin{array}{ccc}
a_{r 1} & 0 & 0 \\
0 & \ddots & 0 \\
0 & 0 & a_{r K}
\end{array}\right],
$$

$$
\mathbf{e}_{|\mathrm{R}| \times 1}=\left[\begin{array}{lll}
1 & \ldots & 1
\end{array}\right]_{1 \times|R|}^{\mathrm{T}} \cdot
$$

Denominator of Formula (14) gives a scalar value. The right side of Formula (14) expresses therefore a vector resulting from a product of 2 matrices and division of this product by a scalar.

A required accuracy $\varepsilon>0$ for estimation of criteria weight values is assumed while estimating weight vector $\mathbf{w}^{(r)}$. A CCM procedure stops when:

$$
\underset{r \in R}{\forall}\left|\mathbf{w}^{(r)}-\mathbf{w}_{-}^{(r)}\right| \leq \boldsymbol{\varepsilon}
$$

where $\mathbf{w}_{-}^{(r)}$ denotes the preceding weight vector $\mathbf{w}^{(r)}$ approximation according to regulatory alternative $r$ and vector $\boldsymbol{\varepsilon}$ defines estimation accuracy:

$$
\boldsymbol{\varepsilon}_{|R| \times 1}=\left[\begin{array}{lll}
\varepsilon & \ldots & \varepsilon
\end{array}\right]_{1 \times|R|}^{\mathrm{T}} .
$$

Adjusted value for the k-th consecutive criterion weight obtained according to the regulatory alternative $r$ is denoted by $\bar{w}_{k}^{(r)}$. Adjusted values of weights are applied for overall evaluation of considered objects:

$$
\mathbf{P}^{(r)}=\overline{\mathbf{A}}^{(r)} \overline{\mathbf{w}}^{(r)} .
$$

We finally obtain the same overall evaluations regardless of applied regulatory alternative:

$$
\overline{\mathbf{P}}=\frac{\mathbf{P}^{(r)}}{\sum_{i=1}^{n} P_{i}^{(r)}} .
$$

Different improvements to are introduced to procedure of criteria weights adjustment. For example Ohya and Kinoshita (2009) proposed to replace a step-wise CCM procedure with the geometric mean concurrent convergence method (GMCCM). They also made an attempt (Ohya and Kinoshita 2011) to endow MDAHP with a mechanism similar to ANP supermatrix (Saaty, 1996). They idea is called super pair-wise comparison matrix (SPCM). 
Merits of DAHP make it an interesting approach for different purposes. One of the most attractive purposes deals with aggregating redundant information coming from different sources.

\section{A sample real estate valuation}

A sample analysis is devoted to agricultural real estate valuation (Dytczak et al., 2009). Three different features are applied for evaluation of real estate objects: agicultural merits $\mathrm{R}$, organisational merits $\mathrm{O}$ and economic merits $\mathrm{E}$. We estimate value for the $\mathrm{N}$ real estate object using known values for 5 real estate objects 14, 13, 4, 3 and 8 . A licensed valuator provided us with knowledge about features of all considered real estate objects. Transactional values for comparative real estate objects are also known. Data for all considered real estate objects are presented in Tab.1.

Table 1. Evaluations of real estate objects (Dytczak et al., 2009)

\begin{tabular}{|c|c|c|c|c|c|c|}
\hline Object & $\mathrm{N}$ & 14 & 13 & 4 & 3 & 8 \\
\hline $\mathrm{R}$ & Weak & Weak & Satisfactory & Satisfactory & Average & Good \\
\hline O & Weak & Weak & Average & Good & Good & Good \\
\hline E & Good & Average & Good & Good & Good & Average \\
\hline Value, PLN/ha & $?$ & 13555 & 19987 & 25402 & 33328 & 39511 \\
\hline
\end{tabular}

Here are the pair-wise comparison judgements of criteria importance and their results (Dytczak et al., 2009):

$$
\mathbf{A}^{(c)}=\left[\begin{array}{ccc}
1 & 5 & 7 \\
\frac{1}{5} & 1 & 2 \\
\frac{1}{7} & \frac{1}{2} & 1
\end{array}\right] \Rightarrow \mathbf{w}=\left[\begin{array}{c}
0.745 \\
0.149 \\
0.106
\end{array}\right]
$$

Pair-wise comparison judgements of real estate objects according to consecutive features and resulting normalised partial evaluations express the following matrices and vectors [2]:

1. For agricultural merits R:

$$
\mathbf{A}^{(\mathrm{R})}=\left[\begin{array}{cccccc}
1 & 1 & \frac{1}{3} & \frac{1}{3} & \frac{1}{7} & \frac{1}{9} \\
1 & 1 & \frac{1}{3} & \frac{1}{3} & \frac{1}{7} & \frac{1}{9} \\
3 & 3 & 1 & 1 & \frac{1}{3} & \frac{1}{5} \\
3 & 3 & 1 & 1 & \frac{1}{3} & \frac{1}{5} \\
7 & 7 & 3 & 3 & 1 & \frac{1}{3} \\
9 & 9 & 5 & 5 & 3 & 1
\end{array}\right] \Rightarrow \mathbf{p}^{(\mathrm{R})}=\left[\begin{array}{l}
0.0386 \\
0.0386 \\
0.1012 \\
0.1012 \\
0.2530 \\
0.4674
\end{array}\right] .
$$

2. For organisational merits $\mathrm{O}$ :

$$
\mathbf{A}^{(0)}=\left[\begin{array}{llllll}
1 & 1 & \frac{1}{3} & \frac{1}{5} & \frac{1}{5} & \frac{1}{5} \\
1 & 1 & \frac{1}{3} & \frac{1}{5} & \frac{1}{5} & \frac{1}{5} \\
3 & 3 & 1 & \frac{1}{3} & \frac{1}{3} & \frac{1}{3} \\
5 & 5 & 3 & 1 & 1 & 1 \\
5 & 5 & 3 & 1 & 1 & 1 \\
5 & 5 & 5 & 1 & 1 & 1
\end{array}\right] \Rightarrow \mathbf{p}^{(0)}=\left[\begin{array}{l}
0.0487 \\
0.0487 \\
0.1103 \\
0.2641 \\
0.2641 \\
0.2641
\end{array}\right] .
$$


3. For economic merits E:

$$
\mathbf{A}^{(\mathrm{E})}=\left[\begin{array}{cccccc}
1 & 5 & 1 & 1 & 1 & 5 \\
\frac{1}{5} & 1 & \frac{1}{5} & \frac{1}{5} & \frac{1}{5} & 1 \\
1 & 5 & 1 & 1 & 1 & 5 \\
1 & 5 & 1 & 1 & 1 & 5 \\
1 & 5 & 1 & 1 & 1 & 5 \\
\frac{1}{5} & 1 & \frac{1}{5} & \frac{1}{5} & \frac{1}{5} & 1
\end{array}\right] \Rightarrow \mathbf{p}^{(\mathrm{E})}=\left[\begin{array}{c}
0.2273 \\
0.0454 \\
0.2273 \\
0.2273 \\
0.2273 \\
0.0454
\end{array}\right]
$$

Consecutive rows and columns of above matrices and vectors correspond to the following sequence of real estate objects: N, 14, 13, 4, 3, 8. All judgement matrices are consistent enough. Overall evaluations $\mathbf{P}$ for considered real estate objects are given by the formula:

$$
\mathbf{P}=\left[\begin{array}{c}
\mathbf{p}^{(\mathrm{R})^{\mathrm{T}}} \\
\mathbf{p}^{(\mathrm{O})^{\mathrm{T}}} \\
\mathbf{p}^{(\mathrm{E})^{\mathrm{T}}}
\end{array}\right] \mathbf{w} .
$$

They are presented together with estimated real estate $\mathrm{N}$ value in Tab.2.

\begin{tabular}{|c|c|c|c|c|c|c|c|c|}
\hline \multicolumn{2}{|c|}{ Criterion } & \multirow{2}{*}{$\mathbf{N}$} & \multirow{2}{*}{14} & \multirow{2}{*}{13} & \multirow{2}{*}{4} & \multirow{2}{*}{3} & \multirow{2}{*}{8} & \multirow{2}{*}{ Sum } \\
\hline & $\mathbf{w}$ & & & & & & & \\
\hline $\mathrm{R}$ & 0.745 & 0.0386 & 0.0386 & 0.1012 & 0.1012 & 0.2530 & 0.4674 & 1 \\
\hline $\mathrm{O}$ & 0.149 & 0.0487 & 0.0487 & 0.1103 & 0.2641 & 0.2641 & 0.2641 & 1 \\
\hline $\mathrm{E}$ & 0.106 & 0.2273 & 0.0454 & 0.2273 & 0.2273 & 0.2273 & 0.0454 & 1 \\
\hline \multicolumn{2}{|c|}{ Evaluation } & 0.058 & 0.041 & 0.115 & 0.140 & 0.252 & 0.394 & 1 \\
\hline \multicolumn{2}{|c|}{$\begin{array}{c}\text { Value, } \\
\text { PLN/ha }\end{array}$} & 15068 & & & & & & \\
\hline
\end{tabular}

Table 2. Data and results for the sample analysis (Dytczak et al., 2009)

Estimated real estate value is obtained thanks to application of linear interpolation of values of nearby real estate objects 14 and 13.

Three real estate objects, namely 14, 4 and 8 are assumed regulatory alternatives next. They pertain to the indices: $r=2,4,6$ respectively. They allow to evaluate criteria in the following way (the assumed order of criteria is: $\mathrm{R}, \mathrm{O}$ and $\mathrm{E}$ ):

1. Relative to the regulatory alternative 14 :

$$
\mathbf{A}^{(2)}=\left[\begin{array}{ccc}
1 & 3 & 1 \\
\frac{1}{3} & 1 & \frac{1}{2} \\
1 & 2 & 1
\end{array}\right] \Rightarrow \mathbf{w}^{(2)}=\left[\begin{array}{l}
0.4429 \\
0.1698 \\
0.3873
\end{array}\right] .
$$

2. Relative to regulatory alternative 4 : 


$$
\mathbf{A}^{(4)}=\left[\begin{array}{lll}
1 & 1 & \frac{1}{2} \\
1 & 1 & 1 \\
2 & 1 & 1
\end{array}\right] \Rightarrow \mathbf{w}^{(4)}=\left[\begin{array}{l}
0.2611 \\
0.3278 \\
0.4111
\end{array}\right]
$$

3. Relative to regulatory alternative 8 :

$$
\mathbf{A}^{(6)}=\left[\begin{array}{lll}
1 & \frac{1}{2} & 1 \\
2 & 1 & 1 \\
1 & 1 & 1
\end{array}\right] \Rightarrow \mathbf{w}^{(6)}=\left[\begin{array}{l}
0.2611 \\
0.4112 \\
0.3278
\end{array}\right] .
$$

Consistency ratio $c . r$. for $\mathbf{A}^{(2)}, \mathbf{A}^{(4)}, \mathbf{A}^{(6)}$ matrices is equal to $0.017,0.047$ and 0.047 respectively.

Accuracy $\varepsilon=0.0001$ is assumed for the CCM procedure. Values of $\bar{w}_{k}^{(r)}$ obtained for consecutive approximation steps are presented in Tab.3. Step zero corresponds to initial vectors $\mathbf{w}^{(2)}, \mathbf{w}^{(4)}, \mathbf{w}^{(6)}(28-30)$. It is evident that 3 steps are enough to obtain stable and consistent criteria weight values.

Table 3. Results of consecutive CCM procedure steps

\begin{tabular}{|c|c|c|c|c|c|c|c|c|c|}
\hline & \multicolumn{7}{|c|}{ Basis for criteria weight estimation - regulatory real estate object } \\
\hline \multirow{2}{*}{ Step } & \multicolumn{3}{|c|}{14} & \multicolumn{3}{|c|}{4} & \multicolumn{3}{|c|}{8} \\
\cline { 2 - 10 } & $\bar{w}_{1}^{(2)}$ & $\bar{w}_{2}^{(2)}$ & $\bar{w}_{3}^{(2)}$ & $\bar{w}_{1}^{(4)}$ & $\bar{w}_{2}^{(4)}$ & $\bar{w}_{3}^{(4)}$ & $\bar{w}_{1}^{(6)}$ & $\bar{w}_{2}^{(6)}$ & $\bar{w}_{3}^{(6)}$ \\
\hline 0 & 0.4429 & 0.1698 & 0.3873 & 0.2611 & 0.3278 & 0.4111 & 0.2611 & 0.4111 & 0.3278 \\
\hline 1 & 0.3016 & 0.1991 & 0.4993 & 0.1923 & 0.2508 & 0.5569 & 0.6037 & 0.2507 & 0.1456 \\
\hline 2 & 0.2751 & 0.1968 & 0.5281 & 0.1637 & 0.2414 & 0.5949 & 0.6708 & 0.2192 & 0.1100 \\
\hline 3 & 0.2735 & 0.1967 & 0.5298 & 0.1618 & 0.2407 & 0.5975 & 0.6747 & 0.2174 & 0.1079 \\
\hline 4 & 0.2735 & 0.1967 & 0.5298 & 0.1618 & 0.2407 & 0.5975 & 0.6747 & 0.2174 & 0.1079 \\
\hline
\end{tabular}

The matrix $\mathbf{A}^{\prime}$ results from vectors $\mathbf{p}^{(\mathrm{R})}, \mathbf{p}^{(\mathrm{O})}, \mathbf{p}^{(\mathrm{E})}$ received from formulae (24-26) and looks as follows:

$$
\mathbf{A}^{\prime}=\left[\begin{array}{lll}
0.0386 & 0.0487 & 0.2273 \\
0.0386 & 0.0487 & 0.0454 \\
0.1012 & 0.1103 & 0.2273 \\
0.1012 & 0.2641 & 0.2273 \\
0.2530 & 0.2641 & 0.2273 \\
0.4674 & 0.2641 & 0.0454
\end{array}\right]
$$

The $\overline{\mathbf{A}}^{(r)}$ matrices $(r=2,4,6)$ that are necessary for estimation of overall evaluations for real estate objects are derived using formula (10). For example matrix $\overline{\mathbf{A}}^{(2)}$ required for the case of the regulatory alternative 14 application looks like this one: 


$$
\overline{\mathbf{A}}^{(2)}=\left[\begin{array}{ccc}
1 & 1 & 5 \\
1 & 1 & 1 \\
2.6224 & 2.2661 & 5 \\
2.6224 & 5.4281 & 5 \\
6.5582 & 5.4281 & 5 \\
12.115 & 5.4281 & 1
\end{array}\right]
$$

Utilisation of the following formula resulting from (19):

$$
\mathbf{P}^{(2)}=\overline{\mathbf{A}}^{(2)} \overline{\mathbf{w}}^{(2)},
$$

where vector $\overline{\mathbf{w}}^{(2)}$ is equal to (see: Tab.3)

$$
\overline{\mathbf{w}}^{(2)}=\left[\begin{array}{lll}
0.2735 & 0.1967 & 0.5298
\end{array}\right]^{\mathrm{T}}
$$

leads to the following vector of overall evaluations for real estate objects:

$$
\mathbf{P}^{(2)}=\left[\begin{array}{llllll}
3.119 & 1 & 3.812 & 4.434 & 5.510 & 4.911
\end{array}\right]^{\mathrm{T}} .
$$

Sum of the above vector components is equal to:

$$
\sum_{i=1}^{n} P_{i}^{(14)}=22.787
$$

Application of formula (20) results therefore in the following overall evaluations for the objects:

$$
\overline{\mathbf{P}}^{(14)}=\left[\begin{array}{llllll}
0.1369 & 0.0439 & 0.1673 & 0.1946 & 0.2418 & 0.2155
\end{array}\right]^{\mathrm{T}} .
$$

Process of estimation of overall real estate evaluations can stop here. We obtain the same normalised overall evaluations for other regulatory alternatives 4 and 8 . For example, we would use the following matrices and vectors according to usage of regulatory alternative 4 :

$$
\overline{\mathbf{A}}^{(4)}=\left[\begin{array}{ccc}
0.3813 & 0.1842 & 1 \\
0.3813 & 0.1842 & 0.2 \\
1 & 0.4175 & 1 \\
1 & 1 & 1 \\
2.501 & 1 & 1 \\
4.620 & 1 & 0.2
\end{array}\right], \quad \overline{\mathbf{w}}^{(4)}=\left[\begin{array}{l}
0.1618 \\
0.2407 \\
0.5975
\end{array}\right],
$$

It proves that the valuated real estate object $\mathrm{N}$ belongs to overall evaluation interval starting with the real estate object 14 and ending with the real estate object 13 . Final value of the real estate object $\mathrm{N}$ is denoted by symbol $C_{\mathrm{N}}$ and is obtained in the same way like in the case of traditional AHP application. Overall evaluations obtained for the real estate objects 14,13 and $\mathrm{N}$ as well as transactional prices for the real estate objects 14 and 13 are applied with this regard: 


$$
C_{\mathrm{N}}=13555+\frac{0.1369-0.0439}{0.1673-0.0439} \cdot(19987-13555)
$$

It is equal to $18402 \mathrm{PLN} / \mathrm{ha}$. This value proves different comparing with the result obtained by means of traditional AHP which applies intuitively estimated evaluation criteria weights.

\section{Conclusions}

Obtained results of MDAHP application for real estate object valuation confirm that a application of regulatory alternatives can influence valuation results. Unlike traditional AHP, however, MDAHP allows to objectivise experience-based knowledge of a licensed evaluator. We therefore can finally obtain valuation results which allow to limit doubts about valuation reliability considerably comparing with the case of traditional AHP utilisation results.

MDAHP is flexible extension of traditional AHP. It seems that it can prove useful even well beyond the application scope expected by its creators. It should be therefore considered a decision support tool extending richness of multi-criteria decision analysis methods essentially.

\section{REFERENCES}

Dytczak, M., Ginda, G., \& Szpringier, M. (2009). Multi-attribute valuation of real estate objects with intangibles, Zeszyty Naukowe WSB we Wroctawiu, 11(11), 275-293.

Kinoshita, E., Nakanishi, M. (1999). Proposal of new AHP model in light of dominant relationship among alternatives, Journal of Operations Research Society of Japan, 42(2), 180-197.

Kinoshita, E., Sekitani, K., Shi, J. (2002). Mathematical properties of Dominant AHP and Concurrent Convergence Method, Journal of the Operations Research Society of Japan, 45(2), 198-213.

Ohya, T., Kinoshita, E. (2011). Super Pairwise Comparison Matrix in the Multiple Dominant AHP, in: Watada J. et al. (Eds.): Intelligent Decision Technologies, 1 - SIST 15, 319-327.

Ohya, T., Kinoshita, E. (2009). The geometric mean concurrent convergence method, in: Proceedings of the 10th International Symposium on the Analytic Hierarchy Process 2009, Pittsburgh, 30 July2 August, Paper No.58.

Saaty, T.L. (1980). Analytic Hierarchy Process. Planning, Priority Setting, Resource Allocation. New York: McGraw-Hill.

Saaty, T.L. (1996). Analytic Network Process. Decision making with feedback. Pittsburgh: RWS Publishing. 